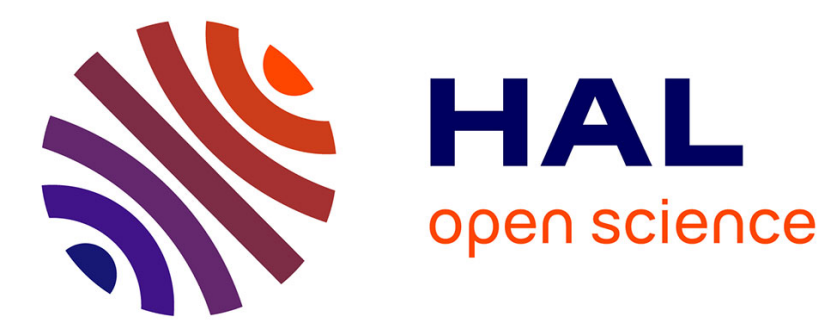

\title{
$\alpha$-Oxygenated Crotyltitanium and Dyotropic Rearrangement in the Total Synthesis of Discodermolide
}

Elsa De lemos, François-Hugues Porée, Alain Commerçon, Jean-François

Betzer, Ange Pancrazi, Janick Ardisson

\section{- To cite this version:}

Elsa De lemos, François-Hugues Porée, Alain Commerçon, Jean-François Betzer, Ange Pancrazi, et al.. $\alpha$-Oxygenated Crotyltitanium and Dyotropic Rearrangement in the Total Synthesis of Discodermolide. Angewandte Chemie International Edition, 2007, 46 (11), pp.1917-1921. 10.1002/anie.200604629 . hal-03333683

\section{HAL Id: hal-03333683 \\ https://hal.science/hal-03333683}

Submitted on 3 Sep 2021

HAL is a multi-disciplinary open access archive for the deposit and dissemination of scientific research documents, whether they are published or not. The documents may come from teaching and research institutions in France or abroad, or from public or private research centers.
L'archive ouverte pluridisciplinaire HAL, est destinée au dépôt et à la diffusion de documents scientifiques de niveau recherche, publiés ou non, émanant des établissements d'enseignement et de recherche français ou étrangers, des laboratoires publics ou privés. 
DOI: 10.1002/anie.200123456

\section{$\alpha$-Oxygenated Crotyltitanium and Dyotropic \\ Rearrangement in Total Synthesis of Discodermolide $^{* *}$}

\section{Elsa de Lemos, François-Hugues Porée, Alain Commerçon, Jean-François Betzer, ${ }^{*}$ Ange Pancrazi, Janick Ardisson*}

Discodermolide is a polyketide natural product that was first isolated in 1990 from extracts of the rare Carribean marine sponge Discodermia dissoluta by Gunasekera. ${ }^{[1]}$ This compound has been shown to inhibit the proliferation of cells by arresting the $G_{2} / M$ phase of the cell cycle. ${ }^{[2]}$ Discodermolide belongs to the class of antimitotic agents known to act by microtubule stabilisation whose clinically used members are Taxol and Taxotere. This product is more potent in stabilizing microtubules, more water soluble than taxoid compounds and exhibits synergy with Taxol. ${ }^{[3]}$

The marine sponge cannot provide the quantities of discodermolide needed for drug development. Therefore, efficient and highly convergent syntheses are needed, and work in the area is increasing. ${ }^{[4]}$

In this paper, we report a new total synthesis of discodermolide which relies upon three particular points: elaboration of syn-anti stereotriads linked to an (Z)-O-enecarbamate group, direct transformation of this core into the terminal (Z)-diene, and stereocontrolled generation of the $(Z)$-trisubstituted double bond by dyotropic rearrangement. Our convergent synthetic strategy, outlined in Scheme 1, relies on the preparation of three subunits $\mathrm{C1}$ C7 2, C8-C14 3 and C15-C24 4. ${ }^{[4 \mathrm{~h}]}$

[*] E. de Lemos, F.-H. Porée, J.-F. Betzer, A. Pancrazi, J. Ardisson Université de Cergy-Pontoise CNRS UMR 8123

5, Mail Gay Lussac - 95031 Cergy Pontoise Cedex Fax: (+33) 1-34-25-73-81

E-mail: janick.ardisson@u-cergy.fr

A. Commerçon

Département des Sciences Chimiques, Chimie des Produits Naturels

Sanofi-Aventis, Centre de Recherche de Vitry-Alfortville 13, Quai Jules Guesde, 94403 Vitry-sur-Seine Cedex, France E-mail: alain.commercon@sanofi-aventis.com

[**] The authors thank Sanofi-Aventis for a fellowship for E.d. L. Technical support from Paris XI University (Châtenay-Malabry and Orsay centers) is gratefully acknowledged (elemental analysis and HRMS).

Supporting information for this article is available on the WWW under http://www.angewandte.org or from the author.

Supporting information for this article is available on the WWW under http://www.angewandte.org or from the author.
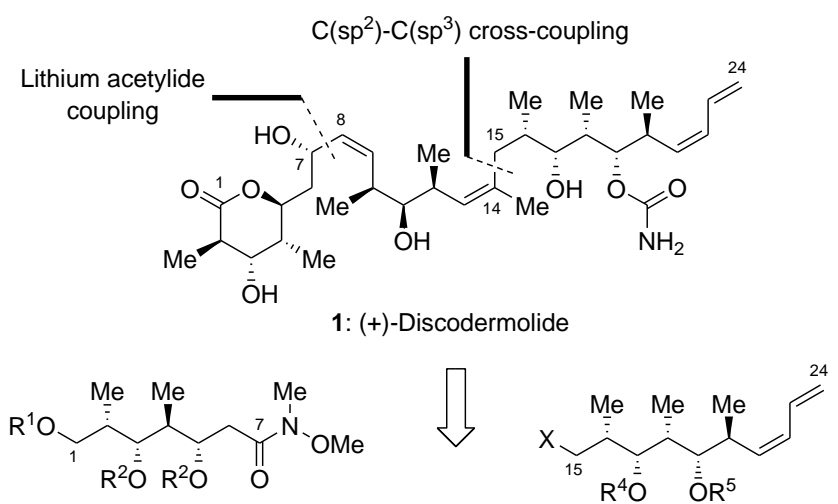

2: C1-C7 subunit

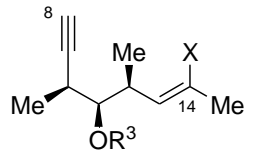

4: C15-C24 subunit

$\mathrm{X}=$ halogen or metal

3: $\mathrm{C} 8-\mathrm{C} 14$ subunit

Scheme 1. Retrosynthetic analysis of discodermolide (1)

Each subunit, or their precursors, possesses a syn-anti methylhydroxyl-methyl triad (in $\mathrm{C} 2-\mathrm{C} 4, \mathrm{C} 12-\mathrm{C} 10$ and $\mathrm{C} 18-\mathrm{C} 20$ respectively for fragments $\mathbf{2}, \mathbf{3}$ and $\mathbf{4}$ ) with adjacent unsaturation (Scheme 2).

Therefore, we developed a convenient and scaleable elaboration of this core motif. A crotyltitanation reaction, developped by Hoppe $^{[5]}$ and widely used in total synthesis by us ${ }^{[6]}$ was selected.

The enantioenriched (R)- $\alpha$-( $N, N$-diisopropylcarbamoyloxy)crotyltitanium $\mathbf{5}$ was easily prepared in situ from crotyl diisopropylcarbamate, an equimolar mixture of $n-\mathrm{BuLi} /(-)$-sparteine and tetra(isopropoxy)titanium. ${ }^{[7]}$ Matched reactions between $\alpha-(S)$ methyl aldehydes 6a-c and $(R)$-crotyltitanium 5 should yield homoaldol adducts 7a-c with high diastereoselectivities. These alcohols possess the three chiral centers in the correct absolute configurations linked to an $(Z)-O$-enecarbamate group.

Moreover, we wanted to take advantage of this (Z)-O enecarbamate function to set up the required unsaturation. The alkyne function in $\mathrm{C} 8-\mathrm{C} 9$ of subunit $\mathbf{3}$ could result from a carbenoid rearrangement, the $\alpha, \beta$-unsaturated Weinreb amide in $\mathrm{C} 7$ of subunit 2 from a cross-metathesis reaction, and the terminal ( $Z$ )-diene in C21-C24 of subunit $\mathbf{4}$ from a nickel-catalyzed cross-coupling reaction. For this strategy, we had to prepare suitable aldehydes for each subunit. 

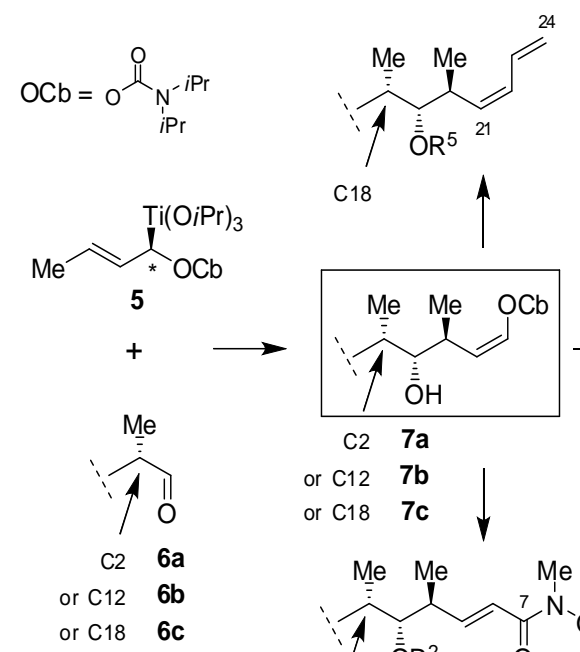

4: C15-C24 subunit

C18
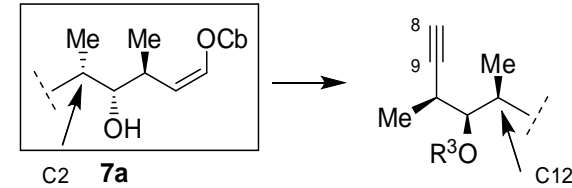

or $\mathrm{C} 12 \mathbf{7 b}$

or $\mathrm{C} 18$

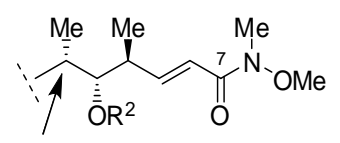

3: C8-C14 subunit

C1-C7 subunit 2 precursor

C2

Scheme 2. Preparation of the syn-anti methyl-hydroxyl-methyl triad cores and subsequent use of the (Z)-O-enecarbamate function.

The crucial central subunit 3 possesses the C13-C14 (Z)trisubstituted double bond and a terminal alkyne. The C8-C9 triple bond should be accessible by an $O$-enecarbamate rearrangement. For stereocontrolled building of the C13-C14 (Z)-trisubstituted functionalized double bond, we considered focusing on a dyotropic rearrangement of 5-lithiodihydrofuran, first examined by Fujisawa ${ }^{[8}$ and developed by Kocienski ${ }^{[9]}$ and us. ${ }^{[10]}$ This attractive reaction should allow the construction of various $(Z)$ - or $(E)$-trisubstituted double bonds, in good yields and with total control of their geometry.

The synthesis of subunit $\mathbf{3}$ began with commercially available bromo alcohol 8 (Scheme 3). A reaction sequence involving cyanide displacement of the bromide, partial reduction of the resulting hydroxy-nitrile compound ${ }^{[11]}$ and direct dehydration provided the optically pure (S)-3-methyl-2,3-dihydrofuran 9 in $64 \%$ overall yield for three steps. The stereochemical control of the C13-C14 (Z)trisubstituted functionalized double bond was based on 1,2-cuprate transfer between the 5-lithio-9 derivative and a cyano-Gilman dimethylcuprate, $\mathrm{Me}_{2} \mathrm{CuLi} \cdot \mathrm{LiCN}$. The use of various additives, and in particular those containing sulphur, allowed us the efficient installation of a methyl group which remained the most difficult to introduce. ${ }^{[12]}$ After tri- $n$-butyltin chloride trapping, the unique $(Z)$ vinyl tin derivative $\mathbf{1 0}$ was obtained in an optimized yield of $68 \%$.

A very large number of oxidation conditions were tested on alcohol 10, but only the use of TEMPO/PhI $(\mathrm{OAc})_{2}$ led to the expected aldehyde $\mathbf{6 b}{ }^{[13]}$ Subsequent allylation of $\mathbf{6 b}$ with the $(R)$ crotyltitanium 5 delivered the pure adduct $7 \mathbf{b}$ in $84 \%$ yield for two steps (no other isomers were detected by NMR analysis). A FritschButtenberg-Wiechell rearrangement allowed the transformation of the $O$-enecarbamate $\mathbf{7 b}$ into the terminal alkyne by treatment with $t \mathrm{BuLi}^{[14]} \mathrm{It}$ is worthy to note that this $\alpha$-elimination reaction, using two equivalents of $t \mathrm{BuLi}$, was faster than the tin/lithium exchange. Subunit 3 was obtained in $26.4 \%$ overall yield for eight steps.
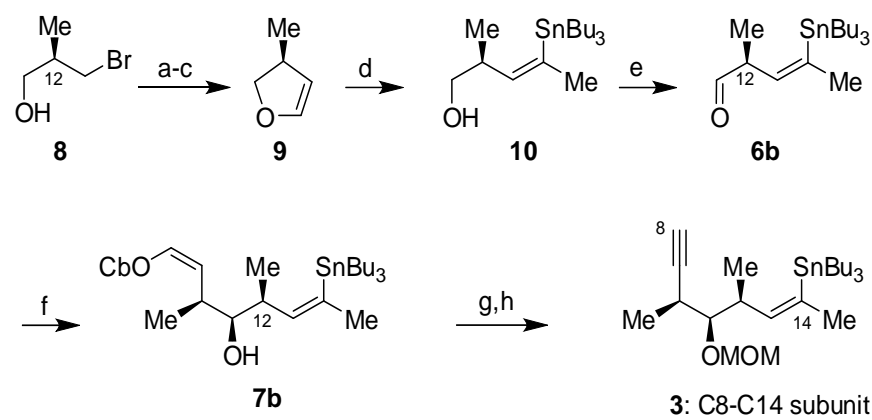

Scheme 3. Synthesis of the C8-C14 subunit (3). a) $\mathrm{NaCN}$, DMSO, $60{ }^{\circ} \mathrm{C}, 24 \mathrm{~h}, 92 \%$; b) DIBAL-H, $\mathrm{CH}_{2} \mathrm{Cl}_{2},-78{ }^{\circ} \mathrm{C}, 2 \mathrm{~h}, 82 \%$; c) $p$-TSA (0.2 mol\%), quinoline, $160 \rightarrow 210^{\circ} \mathrm{C}, 30 \mathrm{~min}, 85 \%$; d) tBuLi,

$\mathrm{Me}_{2} \mathrm{CuLi}$.LiCN, Et ${ }_{2} \mathrm{O} / \mathrm{DMS} 4: 1,0^{\circ} \mathrm{C} \rightarrow \mathrm{RT}, 18 \mathrm{~h}$, then $n \mathrm{Bu}_{3} \mathrm{SnCl}$, $20^{\circ} \mathrm{C} \rightarrow \mathrm{RT}, 4 \mathrm{~h}, 68 \%$; e) TEMPO (10 mol\%), Phl(OAc) $)_{2}, \mathrm{CH}_{2} \mathrm{Cl}_{2}, \mathrm{RT}$, $2 \mathrm{~h}$; f) 5 , pentane/cyclohexane, $-78^{\circ} \mathrm{C}, 3 \mathrm{~h}, 84 \%$ (two steps); g) tBuLi, $\mathrm{Et}_{2} \mathrm{O},-40 \rightarrow-20^{\circ} \mathrm{C}, 20 \mathrm{~min}, 78 \%$; h) MOMCl, TBAl, Hunig's base, $\mathrm{CH}_{2} \mathrm{Cl}_{2}, 18 \mathrm{~h}, 92 \%$. $p$-TSA=p-toluenesulfonic acid, DMS=dimethyl sulfide, TEMPO=2,2,6,6-tetramethyl-1-piperidinyloxy, free radical, Hunig's base=diisopropylethylamine, $\mathrm{MOM}=$ methoxymethyl, TBAI=tetra- $n$-butylammonium iodide.

In order to elaborate subunit $\mathbf{2}$, the optically pure aldehyde $\mathbf{6 a}$ was needed (Scheme 4). This compound could be obtained by classical chemistry in three steps from commercially available (2S)3-hydroxy-2-methylpropionic acid methyl ester 11 (Roche ester). ${ }^{[15]}$ The crotyltitanation reaction between aldehyde $\mathbf{6 a}$ and $(R)$ crotyltitanium 5 furnished homoaldol compound $7 \mathbf{a}$ in $66 \%$ yield for two steps with total diastereoselectivity. The $O$-enecarbamate function of 7a has been deoxygenated under reducing conditions using $i$-propylmagnesium chloride and a nickel catalyst. ${ }^{[16]}$ To our surprise, all attempts to transform this terminal alkene into a $\alpha, \beta$ unsaturated Weinreb amide function by cross-metathesis reaction using Grubbs II or Hoveyda catalyst ${ }^{[17]}$ failed and only the starting material was recovered. ${ }^{[18]}$ Therefore, we undertook an oxidation of $O$-enecarbamate 7a with ozone; the resulting aldehyde was used in a Horner-Wadsworth-Emmons reaction with the commercially available diethyl ( $N$-methoxy- $N$-methylcarbamoylmethyl phosphonate to provide the expected $\alpha, \beta$-unsaturated Weinreb amide $\mathbf{1 2}$ as a single stereomer. A hetero-Michael reaction, with benzaldehyde under anionic conditions, introduced the stereocenter in position $\mathrm{C} 5$ and simultaneously protected the $\mathrm{C} 3$ and $\mathrm{C} 5$ hydroxyl functions as a benzylidene group (diastereomeric ratio > 95:5). ${ }^{[19]}$ This sequence resulted in the effective formation of subunit $\mathbf{2}$, in $35.2 \%$ overall yield for seven steps.
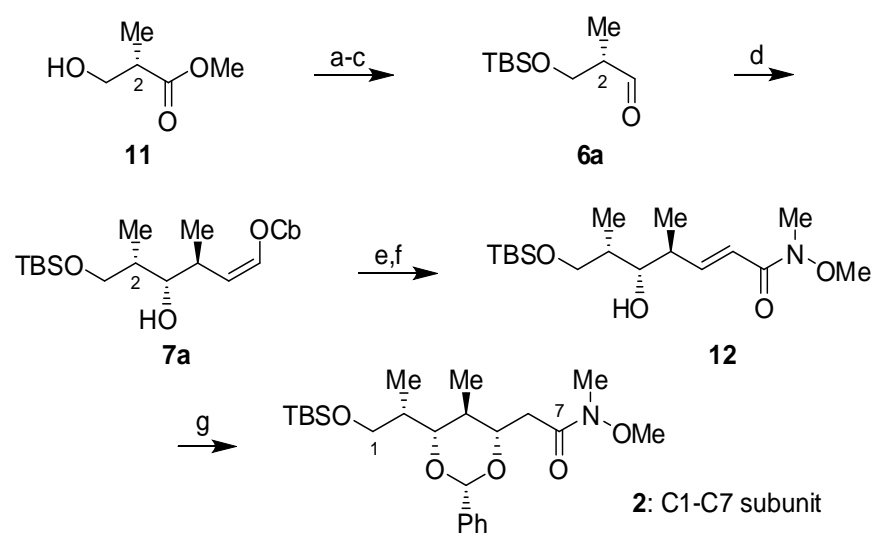
Scheme 4. Synthesis of the $\mathrm{C} 1-\mathrm{C} 7$ subunit (2). a) TBSCl, imid, DMF, $0{ }^{\circ} \mathrm{C} \rightarrow \mathrm{RT}, 18 \mathrm{~h}, 98 \%$; b) DIBAL-H, $\mathrm{CH}_{2} \mathrm{Cl}_{2},-20^{\circ} \mathrm{C}, 30 \mathrm{~min}, 92 \%$; c) $(\mathrm{COCl})_{2}, \mathrm{DMSO}, \mathrm{Et}_{3} \mathrm{~N}, \mathrm{CH}_{2} \mathrm{Cl}_{2},-55^{\circ} \mathrm{C} \rightarrow \mathrm{RT}, 1 \mathrm{~h}$; d) 5,

pentane/cyclohexane, $-78{ }^{\circ} \mathrm{C}, 3 \mathrm{~h}, 66 \%$ (two steps); e) $\mathrm{O}_{3}$, sudan III, $\mathrm{CH}_{2} \mathrm{Cl}_{2},-78{ }^{\circ} \mathrm{C}, 1 \mathrm{~h}$ then $\mathrm{PPh}_{3},-78{ }^{\circ} \mathrm{C} \rightarrow \mathrm{RT}, 3 \mathrm{~h}, 87 \%$; f)

$(\mathrm{EtO}){ }_{2} \mathrm{P}(\mathrm{O}) \mathrm{CH}_{2} \mathrm{C}(\mathrm{O}) \mathrm{NMe}(\mathrm{OMe}), \mathrm{NaH}, \mathrm{THF}, 0{ }^{\circ} \mathrm{C} \rightarrow \mathrm{RT}, 45 \mathrm{~min}, 86 \%$; g) $\mathrm{PhCHO}$, KHMDS, THF, $-20^{\circ} \mathrm{C}, 45 \mathrm{~min}, 79 \%$. TBS=tert-

butyldimethylsilyl, imid=imidazole, DIBAL-H=diisobutylaluminium hydride, KHMDS=potassium bis(trimethylsilyl)amide.

The same Roche ester $\mathbf{1 1}$ was used for the preparation of the third subunit 4 (Scheme 5). This starting material was transformed into aldehyde $\mathbf{1 3}$ by successive protection, reduction and TEMPO/sodium chlorite oxidation. ${ }^{[20]}$ The C16-C18 syn-syn stereotriad was installed by a $\mathrm{BF}_{3} \cdot \mathrm{OEt}_{2}$ catalyzed allylation reaction using achiral tri- $n$-butylcrotylstannane under simple asymmetric induction (diastereomeric ratio was optimized to 95:5). ${ }^{[21]}$. Protection of the $\mathrm{C} 17$ hydroxyl group allowed the isolation of the expected major isomer 14 (74\% yield for two steps). An oxidative cleavage of this alkene then delivered the desired aldehyde $\mathbf{6 c}$.

In a single step, both the C18-C20 syn-anti stereotriad and the (Z)-C21-C22 double bond were set up by an allylation reaction of $\mathbf{6 c}$ with $(R)$-crotyltitanium $\mathbf{5}$. Therefore, carbamate $\mathbf{7} \mathbf{c}$ was elaborated in $77 \%$ yield and total selectivity from alkene 14. Protection of the C19 hydroxyl group and subsequent direct vinylation by a cross-coupling $\mathrm{Ni}(\mathrm{acac})_{2}$ catalyzed reaction ${ }^{[22]}$ between the (Z)-O-enecarbamate moiety and commercially available vinylmagnesium bromide furnished the (Z)-diene $\mathbf{1 5}$ in $85 \%$ yield. ${ }^{[23]}$ After regeneration of the C15 primary hydroxyl group, the clean transformation into alkyl iodide 4 was realised under optimized Garegg conditions in a benzene/diethyl ether mixture under high dilution. ${ }^{[24]}$ In this way, the final subunit $\mathbf{4}$ bearing five stereocenters and the terminal diene was obtained in $16.5 \%$ overall yield for eleven steps.

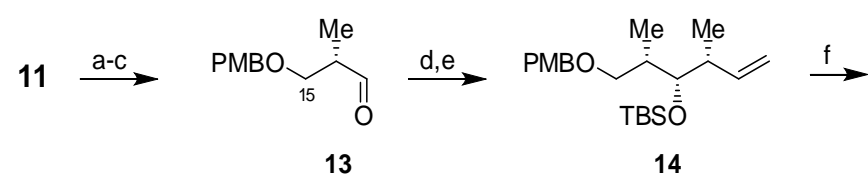

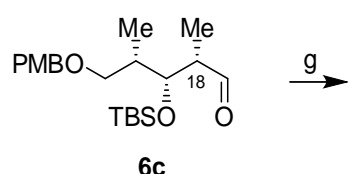

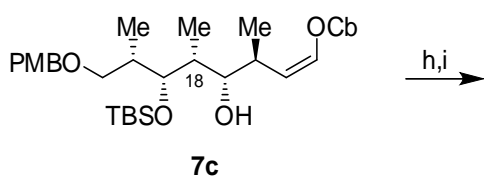

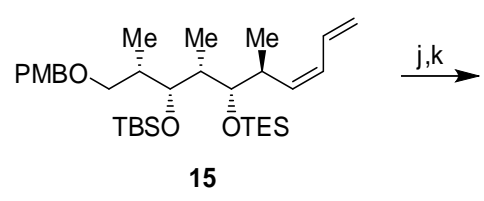

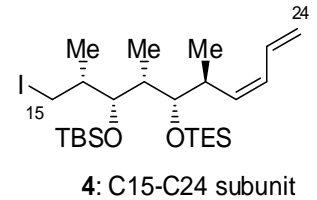

Scheme 5. Synthesis of the C15-C24 subunit (4). a) 1. $\mathrm{CCl}_{3} \mathrm{C}(\mathrm{N}) \mathrm{OPMB}$, PPTS (5 mol\%), $\mathrm{CH}_{2} \mathrm{Cl}_{2} /$ cyclohexane, $20^{\circ} \mathrm{C}, 40 \mathrm{~h}$, $88 \%$; b) LAH, THF, $0{ }^{\circ} \mathrm{C} \rightarrow \mathrm{RT}, 3 \mathrm{~h}, 95 \%$; c) TEMPO (2 mol\%), $\mathrm{NaOCl}, \mathrm{KBr}, \mathrm{NaHCO}_{3}, \mathrm{CH}_{2} \mathrm{Cl}_{2} / \mathrm{H}_{2} \mathrm{O}$; d) Tri- $n$-butylcrotylstannane, $\mathrm{BF}_{3} . \mathrm{OEt}_{2}, \mathrm{Et}_{2} \mathrm{O},-100^{\circ} \mathrm{C}, 2 \mathrm{~h}, 74 \%$ (two steps); e) TBSOTf, 2,6lutidine, $\mathrm{CH}_{2} \mathrm{Cl}_{2}, 0^{\circ} \mathrm{C} \rightarrow \mathrm{RT}$, $2 \mathrm{~h}, 92 \%$; f) $\mathrm{O}_{3}$, sudan III, $\mathrm{CH}_{2} \mathrm{Cl}_{2} / \mathrm{MeOH}$, $-78^{\circ} \mathrm{C}, 2 \mathrm{~h}$ then $\mathrm{Me}_{2} \mathrm{~S},-78{ }^{\circ} \mathrm{C} \rightarrow \mathrm{RT}, 16 \mathrm{~h}$; g) 5, pentane/cyclohexane, $-78{ }^{\circ} \mathrm{C}, 3 \mathrm{~h}, 77 \%$ (two steps); h) TESOTf, 2,6-lutidine, $\mathrm{CH}_{2} \mathrm{Cl}_{2}, 0{ }^{\circ} \mathrm{C} \rightarrow$ $\mathrm{RT}, 1.5 \mathrm{~h}, 78 \%$; i) vinylmagnesium bromide, $\mathrm{Ni}(\mathrm{acac})_{2}$ (10 mol\%), $\mathrm{Et}_{2} \mathrm{O}, 0{ }^{\circ} \mathrm{C}, 16 \mathrm{~h}, 85 \%$; j) DDQ, $\mathrm{CH}_{2} \mathrm{Cl}_{2} / \mathrm{H}_{2} \mathrm{O}, 0{ }^{\circ} \mathrm{C} \rightarrow \mathrm{RT}, 40 \mathrm{~min}, 72 \%$; k) $\mathrm{I}_{2}, \mathrm{PPh}_{3}$, imid, $\mathrm{C}_{6} \mathrm{H}_{6} / \mathrm{Et}_{2} \mathrm{O}, 0{ }^{\circ} \mathrm{C} \rightarrow \mathrm{RT}, 2 \mathrm{~h}, 79 \%$. $\mathrm{PMB}=p$ methoxybenzyle, PPTS=pyridium $p$-toluenesulfonate, TEMPO=2,2,6,6-tetramethyl-1-piperidinyloxy, free radical, TBS=tertbutyldimethylsilyl, TES=triethylsilyl, acac=acetylacetonate, imid=imidazole.

The first coupling reaction of the subunits was achieved by nucleophilic addition of an organometallic species derived from $\mathbf{3}$ on Weinreb amide 2 (Scheme 6). Reaction of 2 with the lithio-3 derivative, generated by metallation with $t \mathrm{BuLi}$, afforded the ynone 16 in $80 \%$ yield. Reduction of $\mathbf{1 6}$, employing the CBS reagent, allowed generation of the $\mathrm{C} 7$ chiral center (diastereomeric ratio > 95:5). ${ }^{[25]}$ After protection of the $\mathrm{C} 7$ hydroxyl group, regeneration of the primary alcohol at $\mathrm{C} 1$ and oxidation under TEMPO/PhI(OAc) conditions, the crude aldehyde was treated with sodium chlorite ${ }^{[26]}$ to afford a carboxylic acid, readily converted into its methyl ester employing TMS-diazomethane. ${ }^{[27]}$ The partial reduction of the triple bond at $\mathrm{C} 8-\mathrm{C} 9$ was realised with $\mathrm{PtO}_{2} \cdot{ }^{[28]}$ Finally, iododestannylation gave the required vinyl iodide $\mathbf{1 8}$ in $30.6 \%$ overall yield for nine steps.

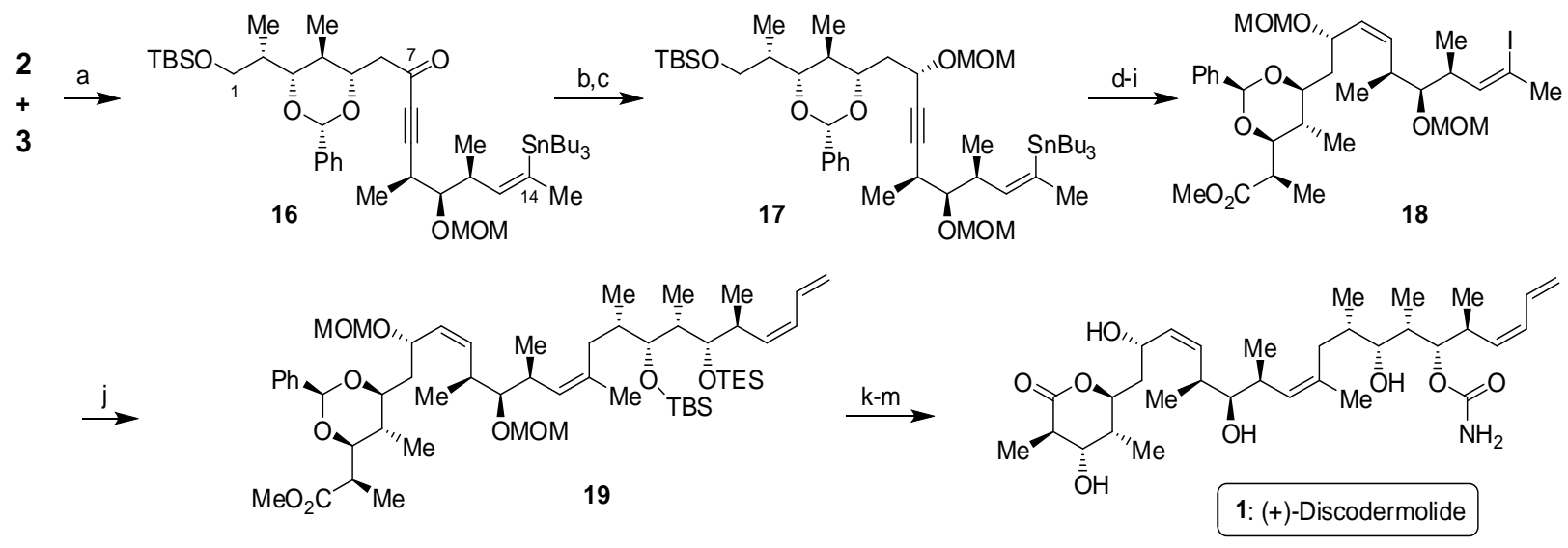


Scheme 6. Coupling reactions and total synthesis of (+)-discodermolide (1). a) 3, $\mathrm{BBuLi}^{\mathrm{Et}} \mathrm{Et}_{2} \mathrm{O},-78{ }^{\circ} \mathrm{C}, 30 \mathrm{~min}$, then $2,0{ }^{\circ} \mathrm{C}, 3 \mathrm{~h}, 80 \%$; b) $(\mathrm{S})-(-)-2-$ Methyl-CBS-oxazaborolidine, $\mathrm{BH}_{3} \cdot \mathrm{Me}_{2} \mathrm{~S}$, THF, $-30{ }^{\circ} \mathrm{C}, 2 \mathrm{~h}, 95 \%$; c) MOMCl, TBAl, Hunig's base, $\mathrm{CH}_{2} \mathrm{Cl}_{2}, \mathrm{RT}, 18 \mathrm{~h}, 86 \%$; d) HF/py, py/THF, RT, 4 h, 96\%; e) TEMPO (10 mol\%), Phl(OAc) $)_{2} \mathrm{CH}_{2} \mathrm{Cl}_{2}, \mathrm{RT}, 3 \mathrm{~h}$; f) $\mathrm{NaClO}_{2}, \mathrm{NaH}_{2} \mathrm{PO}_{4}$, 2-methyl-but-2-ene, $t \mathrm{BuOH} / \mathrm{H}_{2} \mathrm{O}, \mathrm{RT}, 1$ h; g) TMSCHN $\mathrm{C}_{6} \mathrm{H}_{6} / \mathrm{MeOH}, \mathrm{RT}, 15 \mathrm{~min}, 66 \%$ (three steps); h) $\mathrm{H}_{2}, \mathrm{PtO}_{2}$ (30 mol\%), AcOEt, RT, $12 \mathrm{~h}$; i) $\mathrm{I}_{2}, \mathrm{CH}_{2} \mathrm{Cl}_{2}, 0^{\circ} \mathrm{C}, 10 \mathrm{~min}, 71 \%$ (two steps); j) 4, tBuLi, $\mathrm{Et}_{2} \mathrm{O},-78{ }^{\circ} \mathrm{C}, 5 \mathrm{~min}, \mathrm{~B}$-methoxy-9-BBN, THF, $-78{ }^{\circ} \mathrm{C} \rightarrow \mathrm{RT}$, then 18, $\mathrm{CsCO}_{3}, \mathrm{PdCl}_{2} \mathrm{dppf}, \mathrm{AsPh}_{3}, \mathrm{DMF}, \mathrm{H}_{2} \mathrm{O}, 18 \mathrm{~h}, 60 \%$; $\left.\mathrm{k}\right) \mathrm{p}-\mathrm{TSA}, \mathrm{MeOH}, 0{ }^{\circ} \mathrm{C}, 1$ h, $77 \%$; I) $\mathrm{Cl}_{3} \mathrm{CC}(\mathrm{O}) \mathrm{NCO}, \mathrm{CH}_{2} \mathrm{Cl}_{2}, \mathrm{RT}, 15 \mathrm{~min}$, then $\mathrm{K}_{2} \mathrm{CO}_{3}, \mathrm{MeOH}, \mathrm{RT}, 1.5 \mathrm{~h}, 64 \%$; m) HCl 4N, THF, RT, $72 \mathrm{~h}, 70 \%$. CBS= Corey-BakshiShibata, Hunig's base=diisopropylethylamine, MOM=methoxymethyl, TBAl=tetra- $n$-butylammonium iodide, py=pyridine, TMS=trimethylsilyl, $\mathrm{BBN}=$ borabicyclo[3,3,1]nonane, $\mathrm{dppf}=($ diphenylphosphino)ferrocene, $p$-TSA $=p$-toluenesulfonic acid,

The $\sigma$-bond between the major fragment 18 and subunit $\mathbf{4}$ was formed by a $B$-alkyl Suzuki-Miyaura cross-coupling reaction. ${ }^{[29]}$ The reactive trialkyl boronate species, prepared from alkyl iodide 4 with $t \mathrm{BuLi}$ and $B$-methoxy-9-BBN, reacted with 18 under $\mathrm{Pd}(\mathrm{dppf}) \mathrm{Cl}_{2}$ and $\mathrm{AsPh}_{3}$ conditions, ${ }^{[30]}$ to give the fully elaborated discodermolide carbon skeleton 19 in $60 \%$ yield.

After cleavage of the C19 triethylsilyl group and installation of the carbamate moiety ${ }^{[31]}$ the final global deprotection of all protecting groups with concomitant lactonisation, was performed in a $4 \mathrm{~N} \mathrm{HCl}$ solution in THF. Purification of the crude product by flash chromatography $\quad\left(\mathrm{CH}_{2} \mathrm{Cl}_{2} / \mathrm{MeOH} \quad 80: 20\right)$ afforded (+)discodermolide 1 in $70 \%$ yield.

The spectroscopic data observed for our synthetic (+)discodermolide ( ${ }^{1} \mathrm{H}$ NMR, ${ }^{13} \mathrm{C}$ NMR and HRMS) were identical in all respects with those reported for the natural material or another synthetic discodermolide. The specific optical rotation, $[\alpha]_{\mathrm{D}}^{20}+16.2$ $(c=1.0, \mathrm{MeOH})$, was very close to previously reported values. In addition, in vitro cytotoxicity levels comparable to literature data ${ }^{[1,2]}$ were obtained.

In conclusion, total synthesis of discodermolide was achieved in twenty one linear steps with $1.6 \%$ overall yield from commercially available compounds (8 or $\mathbf{1 1})$.

We have shown homoallylic $(Z)-O$-enecarbamate alcohols to be powerful tools in the total synthesis of multi-functional compounds. Allylation reaction of $\alpha-(S)$-methyl aldehydes 6a-c with enantioenriched $(R)$ - $\alpha$-oxygenated crotyltitanium $\mathbf{5}$, conveniently prepared in situ, ensured the stereocontrolled elaboration, for each subunit, of syn-anti methyl-hydroxyl-methyl triads connected to a (Z)-O-enecarbamate. This particular group allowed a direct and easy access to either triple bond or terminal $(Z)$-diene functions.

This efficient and versatile synthesis delivered the natural product and we are currently working to extend this methodology to the preparation of original unnatural discodermolide analogues.

\section{Experimental Section}

((Experimental Details))

Received: ((will be filled in by the editorial staff))

Published online on ((will be filled in by the editorial staff))

Keywords: total synthesis - natural products - allylation - titanium metalation

[1] a) S.P. Gunasekera, M. Gunasekera, R.E. Longley, G.K. Schulte, J. Org. Chem. 1990, 55, 4912-4915; b) S.P. Gunasekera, M. Gunasekera, R.E. Longley, G.K. Schulte, J.
Org. Chem. 1991, 56, 1346; c) S.P. Gunasekera, G.K. Paul, R.E. Longley, R.A. Isbruker, S.A. Pomponi, J. Nat. Prod. 2002, 65, 1643-1648.

[2] Major biological studies reports: a) V.M. Sánchez-Pedregal, K. Kubicek, J. Meiler, I. Lyothier, I. Paterson, T. Carlomagno, Angew. Chem. 2006, 118, 7548-7554; Angew. Chem. Int. Ed. 2006, 45, 7388-7394; b) S. Xia, C.S. Kenesky, P.V. Rucker, A.B. Smith, III, G.A. Orr, S.B. Horwitz, Biochemistry 2006, 45, 39, $11762-11775$; c) S. Honore, K. Kamath, D. Braguer, L. Wilson, C. Briand, M.A. Jordan, Mol. Cancer Ther. 2003, 2, 1303-1311; d) L. He, G.A. Orr, S.B. Horwitz, Drug Discovery Today 2001, 6, 1153-1164; e) L. He, H.Y. Chia-Ping, S.B. Horwitz, Mol. Cancer Ther. 2001, 1, 3-10; f) L.A. Martello, M.J. LaMarche, L. He, T.J. Beauchamp, A.B. Smith, III.; S.B. Horwitz, Chem. Biol. 2001, 8, 843-855; g) M. Kalesse, ChemBioChem. 2000, 1, 171-175; h) E. ter Haar, R.J. Kowalski, E. Hamel, C.M. Lin, R.E. Longley, S.P. Gunasekera, H.S. Rosenkranz, B.W. Day, Biochemistry 1996, 35, 243-250; i) R. Balachandran, E. ter Haar, M.J. Welsh, S.G. Grant, B.W. Day, Anti-Cancer Drugs 1998, 9, 67-76.

[3] a) G.S. Huang, L. Lopez-Barcons, B.S. Freeze, A.B. Smith, III, G.L. Goldberg, S.B. Horwitz, H.M. McDaid, Clin. Cancer. Res. 2006, 12, 298-304; b) L.A. Martello, H.M. McDaid, D.L. Regl, C.-P.H. Yang, D. Meng, T.R.R. Pettus, M.D. Kaufman, H. Arimoto, S.J. Danishefsky, A.B. Smith, III, S.B. Horwitz, Clin. Cancer. Res. 2000, 6, 1978-1987.

[4] Major total synthesis publications: a) J.B. Nerenberg, D.T. Hung, P.K. Somers, S.L. Schreiber, J. Am. Chem. Soc. 1993, 115, 12621-12622; b) D.T. Hung, J.B. Nerenberg, S.L. Schreiber, J. Am. Chem. Soc. 1996, 118, 11054-11080; c) A.B. Smith, III, Y. Qiu, D.R. Jones, K. Kobayashi, J. Am. Chem. Soc. 1995, 117, 12011-12012; d) A.B. Smith, III, T.J. Beauchamp, M.J. LaMarche, M.D. Kaufman, Y. Qu, H. Arimoto, D.R. Jones, K. Kobayashi, J. Am. Chem. Soc. 2000, 122, 8654-8664, and references therein; e) S.S. Harried, G. Yang, M.A. Strawn, D.C. Myles, J. Org. Chem. 1997, 62, 6098-6099; f) S.S. Harried, C.P. Lee, G. Yang, T.I.H. Lee, D.C Myles, J. Org. Chem. 2003, 68, 6646-6660; g) J.A. Marshall, Z.-H. Lu, B.A. Johns, J. Org. Chem. 1998, 63, 817-823; h) J.A. Marshall, B.A. Johns, J. Org. Chem. 1998, 63, 7885-7892; i) I. Paterson, G.J. Florence, K. Gerlach, J.P. Scott, Angew. Chem. 2000, 112, 385-388; Angew. Chem. Int. Ed. 2000, 39, 377-380; j) I. Paterson, G.J. Florence, Eur. J. Org. Chem. 2003, 2193-2208; k) I. Paterson, I. Lyothier, J. Org. Chem. 2005, 70, 5494-5507, and references therein; I) S.J. Mickel, D. Niederer, R. Daeffler, A. Osmani, E. Kuesters, E. Schmid, K. Schaer, R. Gamboni, W. Chen, E. Loeser, F.R. Kinder, Jr., K. Konigsberger, K. Prasad, T.M. Ramsey, O. Repič, R.-M. Wang, G. Florence, I. Lyothier, I. Paterson, Org. Process Res. Dev. 2004, 8, 122-130, and four precedent papers; m) S.J. Mickel, R. Daeffler, W. Prikoszovich, Org. Process Res. Dev. 2005, 9, 113-120; n) O. Loiseleur, G. Koch, J. Cercus, F. Schürch, Org. Process Res. Dev. 2005, 9, 259-271; o) A. Arefolov, J.S. Panek, J. Am. Chem. Soc. 2005, 127, 5596-5603, and references therein.

[5] a) D. Hoppe, Angew. Chem. 1984, 96, 930-946; Angew. Chem., Int. Ed. Engl. 1984, 23, 932-948; b) D. Hoppe, O. Zschage, Angew. Chem. 1989, 101, 67-69; Angew. Chem., Int. Ed. Engl. 1989, 28, 69-71; c) D. Hoppe, O. Zschage, Tetrahedron 1992, 48, 8389-8392.

[6] a) V. Fargeas, P. Le Ménez, I. Berque, J. Ardisson, A. Pancrazi, Tetrahedron 1996, 52, 6613-6634; b) I. Berque, P. Le Ménez, P. Razon, C. Anies, A. Pancrazi, J. Ardisson, A. Neuman, T. Prangé, J.-D. Brion, Synlett 1998, 1132-1134; c) I. Berque, P. 
Le Ménez, P. Razon, A. Pancrazi, J. Ardisson, J.-D. Brion, Synlett 1998, 1135-1137; d) I. Berque, P. Le Ménez, P. Razon, J. Mahuteau, J.-P. Férézou, A. Pancrazi, J. Ardisson, J.-D. Brion, J. Org. Chem. 1999, 64, 373-381.

[7] D. Hoppe, T. Hense, Angew. Chem. 1997, 109, 2376-2410; Angew. Chem. Int. Ed. 1997, 36, 2282-2316.

[8] T. Fujisawa, Y. Kurita, M. Kawashima, T. Sato, Chem. Lett. 1982, 1641-1642.

[9] a) P. Kocienski, S. Wadman, K. Cooper, J. Am. Chem. Soc 1989, 111, 2363-2365; b) P. Kocienski, C. Barber, Pure Appl. Chem. 1990, 62, 1933-1940.

[10] a) P. Le Ménez, V. Fargeas, I. Berque, J. Poisson, J. Ardisson, J.-Y. Lallemand, A. Pancrazi, J. Org. Chem. 1995, 60, 3592 3599; b) V. Fargeas, P. Le Ménez, I. Berque, J. Ardisson, A. Pancrazi, Tetrahedron 1996, 52, 6613-6634.

[11] M.A. Evans, J.P. Morken, Org. Lett. 2005, 7, 3371-3373.

[12] a) E. de Lemos, F.-H. Porée, J.-F. Betzer, A. Pancrazi, J. Ardisson, unpublished results; b) K. Jarowicki, P. Kocienski, S. Norris, M. O'Shea, M. Stocks, Synthesis 1995, 195-198; c) P. Ashworth, B. Broadbelt, P. Jankowski, P. Kocienski, A. Pimm, R. Bell, Synthesis 1995, 199-206; d) A. Pommier, V. Stepanenko, K. Jarowicki, P.J. Kocienski, J. Org. Chem. 2003, 68, 40084013.

[13] A. De Mico, R. Margarita, L. Parlanti, A. Vescovi, G. Piancatelli, J. Org. Chem. 1997, 62, 6974-6977.

[14] a) P. Fritsch, Liebigs Ann. Chem. 1894, 279, 319-323; b) W.P. Buttenberg, Liebigs Ann. Chem. 1894, 279, 324-337; c) H. Wiechell, Liebigs Ann. Chem. 1894, 279, 337-344; For reviews see : d) M. Braun, Angew. Chem. 1998, 110, 444-465; Angew. Chem. Int. Ed. 1998, 37, 430-451; e) R. Knorr, Chem. Rev. 2004, 104, 3795-3849.

[15] W.R. Roush, A.D. Palkowitz, K. Ando, J. Am. Chem. Soc. 1990, 112, 6348-6359.

[16] a) P. Kocienski, N.J. Dixon, Synlett 1989, 52-54; b) R.W. Hoffmann, V. Giesen, M. Fuest, Liebigs Ann. Chem. 1993, 629639.

[17] a) A. Fürstner, Angew. Chem. 2000, 112, 3140-3172; Angew. Chem. Int. Ed. 2000, 39, 3012-3043; b) J. Cossy, S. BouzBouz,
A.H. Hoveyda, J. Organomet. Chem. 2001, 634, 216-221; c) T.L. Choi, A.K. Chatterjee, R.H. Grubbs, Angew. Chem. 2001, 113, 1317-1319; Angew. Chem. Int. Ed. 2001, 40, 1277-1279.

[18] Direct attempts carried out on compound 7a failed.

[19] J.A. Gauchet-Prunet, D.A. Evans, J. Org. Chem. 1993, 58, 2446-2453.

[20] P.L. Anelli, C. Biffi, F. Montanari, S. Quici, J. Org. Chem. 1987, 52, 2559-2562.

[21] a) G.E. Keck, D.E. Abbott, Tetrahedron Lett. 1984, 25, 1883 1886; b) Y. Yamamoto, H. Yatagai, Y. Ishihara, N. Maeda, K. Maruyama, Tetrahedron 1984, 40, 2239-2246; c) G.E. Keck, K.A. Savin, N.K. Cressman, D.E. Abbott, J. Org. Chem. 1994, 59, 7889-7896.

[22] a) E. Wenkert, E.L. Michelotti, C.S. Swindell, J. Am. Chem. Soc. 1979, 101, 2246-2247; b) E. Wenkert, E.L. Michelotti, C.S. Swindell, M. Tingoli, J. Org. Chem. 1984, 49, 4894-4899.

[23] F.-H. Porée, A. Clavel, J.-F. Betzer, A. Pancrazi, J. Ardisson, Tetrahedron Lett. 2003, 44, 7553-7556.

[24] a) P.J. Garegg, B. Samuelsson, J. Chem. Soc., Perkin Trans. 1 1980, 2866-2869; b) E.J. Corey, S.G. Pyne, W.-G. Su, Tetrahedron Lett. 1983, 24, 4883-4886.

[25] a) E.J. Corey, R.K. Bakshi, S. Shibata, J. Am. Chem. Soc. 1987, 109, 5551-5553; b) E.J. Corey, C.J. Helal, Angew. Chem. 1998, 110, 2092-2118; Angew. Chem. Int. Ed. 1998, 37, 1986-2012.

[26] E. Dalcanale, F. Montanari, J. Org. Chem. 1986, 51, 567-569.

[27] a) N. Hashimoto, T. Aoyama, T. Shioiri, Chem. Pharm. Bull. 1981, 29, 1475-1478; b) Y. Hirai, T. Aida, S. Inoue, J. Am. Chem. Soc. 1989, 111, 3062-3063.

[28] L.F. Tietze, J. Görlitzer, A. Schuffenhauer, M. Hübner, Eur. J. Org. Chem. 1999, 1075-1084.

[29] S.R. Chemler, D. Trauner, S.J. Danishefsky, Angew. Chem. 2001, 113, 4676-4701; Angew. Chem. Int. Ed. 2001, 40, 45444568.

[30] J.A. Marshall, G.M. Schaaf, J. Org. Chem. 2003, 68, 7428-7432.

[31] P. Kočovský, Tetrahedron Lett. 1986, 27, 5521-5524. 
Elsa de Lemos, François-Hugues Porée, Alain Commerçon, JeanFrançois Betzer, ${ }^{*}$ Ange Pancrazi, Janick Ardisson*

$\alpha$-Oxygenated Crotyltitanium and Dyotropic Rearrangement in Total Synthesis of Discodermolide
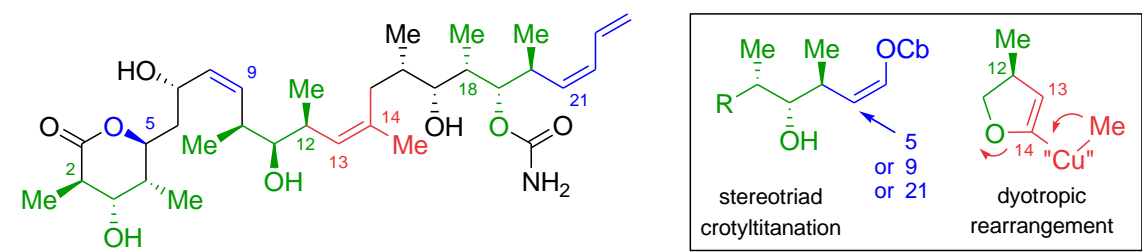

The total synthesis of

discodermolide relied upon three particular points: elaboration of synanti stereotriads linked to a (Z)-Oenecarbamate group, direct transformation into the terminal (Z)diene and stereocontrolled generation of the (Z)-trisubstituted double bond by a dyotropic rearrangement. 